\title{
COMPENSACION AL MENOR TIEMPO DE PASTOREO CON DIFEREN- TES NIVELES DE HENO DE ALFALFA EN ENGORDE DE OVINOS
}

\author{
Amparo Huamán C.', Rosa Dávalos R.², Felipe San Martín H. ${ }^{2}$, \\ Custodio Bojórquez R. ${ }^{1}$, Fernando Carcelén C. ${ }^{2}$ y Alfredo Pérez S. ${ }^{2}$
}

\section{Mtitract}

The impact of variable alfalfa hay feed replacement in fattening sheep during the dry season was evaluated at IVITA's El Mantaro Research Station in the central Andean Mantaro valley. A total of 60 castrated one-year old sheep were grazed on two hectares of rye-grass and clover pasture for 56 days. The sheep were randomly distributed into four groups of 15 each. The control group (T0) grazed 10 hours daily, while the experimental groups grazed 8 (T1), 6 (T2) and 4 (T3) hours daily and received 3, 6 and $9 \mathrm{~kg}$ of alfalfa hay respectively. During the experiment, daily weight gains of $184,167,181$ and 170 grams were registered for groups T0, T1, T2, and T3, with total gains of 10.3 , $9.4,10.1$ and $9.5 \mathrm{~kg}$ respectively. The variation in weight gain under the four regimes was not statistically significant $(\mathrm{P}>0.05$ ). Cost benefit analysis (calculated at $\mathrm{S} . / 3.5 \mathrm{per}$ $\mathrm{kg}$ excluding purchase cost of the sheep) yielded $4.59,4.04,4.24$ and 3.86 for T0 to T3, with profits of $38.8,35.3,38.2$ and $35.9 \%$ respectively. The results demonstrate that alfalfa hay feed replacement compensates reduced grazing time during the dry season.

Key words: Sheep, grazing time, alfalfa hay, cultivated pasture, feeding.

\section{Bretinen}

El uso de heno de alfalfa como reemplazo alimenticio durante la estación seca fue evaluado durante 56 días en dos hectáreas de pasto cultivado en la estación de IVITA El Mantaro. El experimento se diseñó usando 60 ovinos castrados (de un año de edad) distribuidos al azar en cuatro grupos de 15 animales por grupo. El grupo control (T10) fue dejado 10 horas pastoreando en pastos cultivados y los grupos experimentales por 8 (T8); 6 (T6) y 4 (T4) horas, y adicionalmente se les dio 3 (T8); 6 (T6) y 9 (T4) kg de heno de alfalfa. Los incrementos de peso por animal fueron de: $10.3,9.4,10.1$ y $9.5 \mathrm{~kg}$ por animal y la ganancia diaria por animal fue de: $184,167,181$ y $170 \mathrm{~g}$ para los tratamientos T10, T8, T6 y T4, respectivamente, sin mostrar diferencias $(\mathrm{P}>0.05)$. El análisis económico señala un beneficio por animal de $36.1,32.7,35.5$ y 33.3, sin incluir el precio inicial del capón. La relación beneficio - costo fue de 4.59, 4.04, 4.24 y 3.86; y la rentabilidad fue de: $38.8,35.3,38.2$ y $35.9 \%$ para los tratamientos T10, T8, T6 y T4, respectivamente, considerando el precio de $\mathrm{kg}$ de peso vivo de $\mathrm{S} / .3 .5$. Se concluye que la disminución de horas de pastoreo de la asociación rye grass - trébol es totalmente compensado con el heno de alfalfa.

Palabras clave: Ovino, heno de alfalfa, pastura cultivada, horas de pastoreo, alimentación.

\footnotetext{
'Estación Experimental del IVITA - El Mantaro - FMV - UNMSM

${ }^{2}$ FMV - UNMSM. Apdo. 4l-0068
} 
En la sierra central del Perú, existen dos períodos marcados; el período de lluvia que comprende los meses de setiembre a marzo, con abundancia de pasto, y el período de seca de abril a agosto, con escasez de forrajes.

Con la introducción de pastos cultivados en la sierra central, se ha logrado en parte mejorar la eficiencia y rentabilidad de los sistemas productivos. Sin embargo, esta ventaja se ve aún limitada por los cambios climáticos estacionales que también merman la producción de materia seca de los pastos en época de seca. Una de las estrategias para contrarrestar esta disminución de la producción de pasto cultivado es el uso de heno de alfalfa henificado.

El objetivo del estudio fue el de evaluar biológica y económicamente la sustitución de pastos cultivados por heno de alfalfa en períodos de escasez de forraje a través de una reducción de horas de pastoreo y compensado por el suministro de heno de alfalfa.

\section{Vharbles: Vhmo:}

El estudio se realizó en la Estación Experimental IVITA - El Mantaro de la Facultad de Medicina Veterinaria - UNMSM, ubicado en el distrito El Mantaro, provincia de Jauja, departamento de Junín, a una altitud de $3320 \mathrm{~m}$, con una temperatura promedio anual de $12^{\circ} \mathrm{C}$ y una precipitación pluvial promedio anual de $770 \mathrm{~mm}$.

Se dispuso de 2 ha de pastos cultivados (trébol - rye grass), divididos en ocho lotes, permitiendo pastoreos de seis días con descansos de 42 días para cada lote. Se utilizaron 60 ovinos machos castrados, de la raza Junín, con un peso y edad promedio de $26.6 \mathrm{~kg}$ y 1 año, respectivamente. Los animales fueron distribuidos al azar. Se usó el diseño completamente al azar con cuatro tratamientos y 15 repeticiones por tratamiento. Donde los resultados de ganancia de peso por tratamiento fueron sometidos al análisis de varianza y la prueba de DMS (protegida) $(\mathrm{P}<0.05)$, cada uno; a saber:

T10: Diez horas de pastoreo en pasturas cultivadas y $0 \mathrm{~g}$ de heno de alfalfa por animal.

T8: Ocho horas de pastoreo en pasturas cultivadas más $200 \mathrm{~g}$ de heno de alfalfa por animal.

T6: Seis horas de pastoreo en pasturas cultivadas más $400 \mathrm{~g}$ de heno de alfalfa por animal.

T4: Cuatro horas de pastoreo en pasturas cultivadas más $600 \mathrm{~g}$ de heno de alfalfa por animal.

Se estimó la ganancia de peso, medido por la pendiente de la regresión lineal entre peso vivo semanal y días de engorde. También se evaluó el costo de producción y relación beneficio costo.

\section{Remithos Y Dicuston}

\section{Evaluación de los forrajes}

Como se muestra en el Cuadro 1, el contenido proteico tanto del pasto cultivado como del heno fue alfalfa es superior al $18 \%$, considerándose ambos como forrajes de muy buena calidad. (Abrams, 1965).

\section{Ganancia de peso}

Los incrementos de peso por tratamiento fueron similares $(\mathrm{P}>0.05)$ (Cuadro 2). Existe un incremento de peso de cinco a nueve veces mayor durante los primeros 28 días respecto a los últimos 28 días, esta alta ganancia de peso refleja el efecto del crecimiento compensatorio de los animales que estuvieron pastoreando previamente al experimento en pastos naturales. 
Cuadro 1. Composición químico nutricional del heno de alfalfa y pasto cultivado (en porcentaje de base seca).

\begin{tabular}{lcc}
\hline \multicolumn{1}{c}{ Determinación } & Heno de Alfalfa & Pasto cultivado \\
\hline Humedad & 15 & 78.8 \\
Materia Seca & 85 & 21.2 \\
Proteínas & 21.9 & 18.3 \\
Grasas & 3.2 & 3.95 \\
Fibra Cruda & 34.6 & 16.9 \\
Ext. no Nitrog. & 34.0 & 51.95 \\
Cenizas & 6.3 & 8.9
\end{tabular}

* Pasto cultivado compuesto por: $72 \%$ de gramíneas (rye grass inglés e italiano), $18.6 \%$ de leguminosas (trébol blanco y rojo) y $9.4 \%$ de materia muerta.

Cuadro 2. Ganancia de peso dia por tratamiento durante el periodo de engorde.

\begin{tabular}{cccccc}
\hline \multicolumn{9}{c}{ Tratamiento } \\
\hline & $\begin{array}{c}\text { Horas de pastoreo y suplemento diario } \\
\text { con heno de alfalfa }(\mathrm{g} / \mathrm{animal})\end{array}$ & \multicolumn{3}{c}{ Ganancia diaria $(\mathrm{g})$} \\
\cline { 2 - 6 } Clave & Pastoreo $(\mathrm{h})$ & Heno de alfalfa $(\mathrm{g})$ & $0-28$ & $29-56$ & $0-56$ \\
\hline \multirow{2}{*}{ T10 } & 10 & 0 & $373 \mathrm{a}$ & $70 \mathrm{a}$ & $184 \mathrm{a}$ \\
T8 & 8 & 200 & $368 \mathrm{a}$ & $53 \mathrm{a}$ & $167 \mathrm{a}$ \\
T6 & 6 & 400 & $382 \mathrm{a}$ & $53 \mathrm{a}$ & $181 \mathrm{a}$ \\
T4 & 4 & 600 & $377 \mathrm{a}$ & $42 \mathrm{a}$ & $170 \mathrm{a}$ \\
\end{tabular}

* Letras similares en columna indica no diferencia significativa $(\mathrm{P}>0.05)$ a la prueba de DMS (protegida)

Las respuestas similares entre tratamientos se explican a que el heno de alfalfa compensó la disminución de horas de pastoreo de los pastos cultivados (Cuadro 2).

Las ganancias de peso fueron similares a los obtenidos por Coronado et al. (1976); Farfán et al. (1981) y Porras (1977) y superiores a los obtenidos en otros engordes bajo pasturas cultivadas (Farfän et al., 1983; Coronado y Farfán, 1980; Farfän et al., 1981; Thierolf et al., 1981; Bojórquez, 1981; Ortiz, 1981 y Gómez, 1989).

\section{Analisis económico}

El costo de producción de un $\mathrm{kg}$ de peso vivo ganado por ovino y por tratamiento se muestra en el Cuadro 3. La relación beneficio/costo, beneficio total por ovino y la utili- dad neta por ovino se muestra en el Cuadro 4.

El costo de $100 \mathrm{~g}$ de heno de alfalfa (de S/. 0.016), fue ligeramente superior a la hora de pastoreo (S/.0.014); por esta razón, el costo de producción por $\mathrm{kg}$ de peso vivo ganado, se incrementa a medida que se utiliza mayor cantidad de heno de alfalfa, y en forma inversa ocurre con la relación beneficio/costo. Sin embargo, el beneficio total por animal ( $\mathrm{kg}$ ganado multiplicado por el precio de venta $=3.50$ nuevos soles) y la utilidad neta (beneficio total menos el costo de producción), se muestran a favor de T10 y T6 con similares resultados; T10 por obtener el menor costo de producción y la mayor ganancia de peso vivo y T6 por obtener la mejor ganancia de peso dentro de los tratamientos que recibieron heno de alfalfa. 
A. Huamán, et al.

Cuadro 3. Costos de producción en Nuevos Soles (S/.)

\begin{tabular}{cccccc}
\hline Clave & $\begin{array}{c}\text { S/.hora de } \\
\text { pastoreo }\end{array}$ & S/.g heno & $\begin{array}{c}\text { Costo diario de } \\
\text { alimento/ovino }\end{array}$ & $\begin{array}{c}\text { Costo de alimento } \\
\text { por período de } \\
\text { engorde }\end{array}$ & $\begin{array}{c}\text { Costo/kg de } \\
\text { peso vivo } \\
\text { ganado }\end{array}$ \\
\hline T10 & 0.140 & 0 & 0.1400 & 7.840 & 0.523 \\
T8 & 0.112 & 0.0327 & 0.1447 & 8.103 & 0.540 \\
T6 & 0.084 & 0.0654 & 0.1494 & 8.366 & 0.558 \\
T4 & 0.056 & 0.0981 & 0.1541 & 8.630 & 0.575 \\
\hline
\end{tabular}

Cuadro 4. Datos económicos en Nuevos Soles (S/.)

Tratamiento Relación Beneficio/Costo Beneficio Total/Animal* Utilidad Neta**

\begin{tabular}{cccc}
\hline & & & \\
T10 & 6.69 & 36.1 & 32.52 \\
T8 & 4.04 & 32.7 & 30.64 \\
T6 & 4.24 & 35.5 & 32.45 \\
T4 & 3.86 & 33.3 & 29.87 \\
\hline
\end{tabular}

* Kg ganado multiplicado por el precio de venta (S/. 3.50)

** Beneficio total menos costo de producción

La rentabilidad obtenida para los tratamientos T10, T8, T6 y T4 fueron de 38.8, 35.4, 38.2 y $35.9 \%$, respectivamente. Al respecto Bojórquez (1981), reporta rentabilidad de 12.8 $\%$, Coronado y Cornejo (1977) de $22.9 \%$ en un período de 4 meses, y Llacza (1978) de $43 \%$.

Se concluye que el T6 es de igual rentabilidad que el T10, constituyéndose este tratamiento en la primera opción de reemplazo; sin embargo, los tratamientos T8 y T4 son alternativas viables, dependientes de la disponibilidad de pasto cultivado y el heno de alfalfa.

\section{Literatura cinatha}

1. Abrams, J.T. 1965. Nutrición animal y dietética veterinaria, Ed. Acribia, Zaragoza. p. 303-330.

2. Bojórquez, $A$. 1981. Estudio comparativo de la producción de carne en caponcitos
Junín, sometidos a 4 sistemas de explotación. Tesis para optar el Título de Ingeniero Zootecnista. Universidad Nacional Agraria La Molina. Lima. Perú. $97 \mathrm{p}$.

3. Coronado; L. y G Farfán. 1980. Engorde de ovinos Corriedale en pastos cultivados. En: Resúmenes de la III Reunión Científica Anual de la Asociación Peruana de Producción Animal (APPA). Lima. Perú.

4. Coronado, L. y G Cornejo. 1977. Evaluación económica preliminar del engorde de ovinos en pasturas cultivadas. En: An. $5^{\text {a }}$ Cong Nac Ciencias Vet. Perú. Arequipa.

5. Coronado, L.; A. Landa; D. Miles y L. Vaccaro. 1976. Engorde de ovinos en pasturas permanentes. En: Resúmenes $5^{\text {a }}$ Reunión Nacional de Asociación de Especialistas e Investigadores Forrajeros del Perú. Huancayo - Perú. p. 121. 
6. Farfán, G; L. Coronado; C. Bojórquez; H. Acuña y F. Arévalo. 1983. Dos asociaciones de pasto cultivado. En Resum VI Reunion Cient Aun Asoc Peruana Prod Anim. Lambayeque. Perú. AR-8.

7. Farfán, G; L. Coronado; C. Bojórquez y F. Arévalo. 1981. Ganancia de peso de ovinos Corriedale en pastos cultivados. En: Resum IV Reunion Cient Anu Asoc Peruana Prod Anim. Ayacucho. Perú. p. 12.

8. Gómez, F. 1989. Evaluación productiva y económica de la castración e inducción al criptorquidismo en el engorde intensivo de carneros. Tesis para optar el título de Ingenieo Zootecnista. Universidad Nacional Agraria La Molina. Lima. Perú. $92 \mathrm{p}$.

9. Llacza, A. C. 1978. Determinación del óptimo económico en engorde de ovinos de saca tipo Junín, en pasturas cultivadas en la zona altoandina. Tesis para optar el título de Ingeniro Agronomo. Universidad Nacional San Cristóbal de Huamanga. Ayacucho. Perú. $136 \mathrm{p}$.

11.Ortiz, J. 1981. Engorde de ovinos con pastos cultivados. En: Resúmenes de la IV Reunión Científica Anual de la Asoc Peruana Prod Anim.. Ayacucho. Perú.

12.Porras, E. 1977. Comparativo de engorde de borregas de saca con pasturas Nativas y asociada introducida. Tesis para optar el Título de Ingeniero Zootecnista. Universidad Nacional del Centro del Perú. Huancayo. $88 \mathrm{p}$.

13.Thierolf, J.G; H. Castillo y F. Konold. 1981. Pastos cultivados en la sierra y engorde compensatorio de ovinos. Cooperación Técnica en Planificación Peruano - Alemana. Lima. Perú. 85 p. 